\title{
Normal Aging Increases Discriminal Dispersion in Visuospatial Short-Term Memory
}

\author{
Hannes Noack \\ Max Planck Institute for Human Development, Berlin, Germany
}

\author{
Martin Lövdén \\ Max Planck Institute for Human Development, Berlin, Germany, \\ and Karolinska Institutet and Stockholm University, \\ Stockholm, Sweden
}

\author{
Ulman Lindenberger \\ Max Planck Institute for Human Development, Berlin, Germany
}

\begin{abstract}
Computational models of cognitive aging propose that age-related decrements in cognitive performance, including short-term memory (STM), result from less distinct stimulus representations. When applied to visual STM, these models predict higher discriminal dispersion (L. L. Thurstone, 1927, Psychophysical analysis, The American Journal of Psychology, 38, 368-389.) in older adults than in younger adults. To test this prediction, we used a change-detection paradigm for visuospatial locations, with different levels of cognitive load (one, three, or five items) and retention interval (100 or 1,000 ms). Adult age differences were not reliable at Load 1, but were substantial at Loads 3 and 5. Effects of retention time did not differ across age groups, suggesting that age-related differences originated mainly from early processing stages. Applying a mixture model to the data revealed age-related increases in discriminal dispersion and decreases in asymptotic discrimination performance (indexing STM capacity). We concluded that age-related declines in discriminal dispersion, in addition to increasing capacity limitations, impair visual STM performance with advancing adult age.
\end{abstract}

Keywords: aging, visual short-term memory, change detection, discriminal dispersion

Cross-sectional and longitudinal investigations have resulted in a rich database on age-related differences and changes in memory performance (Hoyer \& Verhaeghen, 2006; Park \& Payer, 2006), and theoretical accounts thereof (e.g., Balota, Dolan, \& Duchek, 2000; Light, 1991). One such theoretical proposition is the neural noise hypothesis (Welford, 1981, 1984), which states that the entropy (i.e., uncertainty) of neural signaling increases in aging. That is, more random signals originating from the senses and from neural interactions within the brain lead to more random and less

This article was published Online First May 7, 2012.

Hannes Noack, Center for Lifespan Psychology, Max Planck Institute for Human Development, Berlin, Germany; Martin Lövdén, Center for Lifespan Psychology, Max Planck Institute for Human Development, Berlin, Germany, and Aging Research Center, Karolinska Institutet and Stockholm University, Stockholm, Sweden; and Ulman Lindenberger, Center for Lifespan Psychology, Max Planck Institute for Human Development, Berlin, Germany.

Supported by the Max Planck Society and the Sofja Kovalevskaja Award administered by the Alexander von Humboldt Foundation and donated by the German Federal Ministry for Education and Research (BMBF). We thank Andreas Brandmaier, Florian Schmiedek, Viola Störmer, Manuel Völkle, Timo von Örtzen, and Markus Werkle-Bergner for helpful discussions as well as Elisabeth Wenger, Anja Rehatschek, and Ewgenia Juschkewitsch for collecting the data.

Correspondence concerning this article should be addressed to Hannes Noack, Center for Lifespan Psychology, Max Planck Institute for Human Development, Lentzeallee 94, 14195 Berlin, Germany. E-mail: noack@mpib-berlin.mpg.de precise perceptual and memory representations. At the heart of this proposition is the assumption that representations are probabilistic in nature and may therefore be best characterized by a distribution with a modal value and a "discriminal dispersion"1 (cf. Thurstone, 1927; see also Wilken \& Ma, 2004; Verghese, 2001). Within this framework, higher levels of neural noise are associated with greater discriminal dispersion.

Age-related reductions in signal specificity and, thus, wider and more overlapping receptive fields in old age have been found in the primary somatosensory cortex of rats (Spengler, Godde, \& Dinse, 1995) and in sensory areas of the visual cortex of cats (Hua et al., 2006) and monkeys (Liang et al., 2010; Yang et al., 2008; Yu, Wang, Li, Zhou, \& Leventhal, 2006). Similarly, age-related increases in the ratio of spiking variability to mean spike rate (Yang et al., 2009) and in spontaneous neuronal background activity (Hua et al., 2006) have been found in the visual cortex of monkeys. These results support the notion of a decreasing signalto-noise ratio in the aging brain and thus more dispersed representations.

\footnotetext{
${ }^{1}$ Thurstone introduced the concept of the discriminal processes to describe the "... process by which the organism identifies, distinguishes, discriminates, or reacts to stimuli ..." (1927, p. 368) without specific reference to the nature of the process. Discriminal processes are characterized by discriminal dispersion. We used this classic term because this is what we measured. We made the additional assumption, however, that discriminal dispersion is closely related to the distinctiveness of neural activation patterns.
} 
Neural network models implementing age-related decreases in signal-to-noise ratio through variation of synaptic gain have led to the prediction of less distinct (i.e., more overlapping) representations in the aging brain (Braver \& Barch, 2002; Li, Lindenberger, \& Frensch, 2000; Li, Lindenberger, \& Sikström, 2001; Li \& Sikström, 2002). This prediction of reduced activation specificity (or dedifferentiation) has been validated at macroscopic levels with neuroimaging measures (Grady \& Craik, 2000; Park et al., 2004; Park \& Reuter-Lorenz, 2009). For example, Park et al. (2004) studied activity patterns of the ventral visual cortex and detected areas that responded selectively to the presentation of faces, houses, chairs, or pseudowords in younger participants. In older adults, the distinctiveness of the activation was reduced (see also Carp, Gmeindl, \& Reuter-Lorenz, 2010; Carp, Park, Polk, \& Park, 2011; Goh, Suzuki, \& Park, 2010; Park, Carp, Hebrank, Park, \& Polk, 2010; Payer et al., 2006; Voss et al., 2008).

Focusing on behavioral measures, Allen, Kaufman, Smith, and Propper (1998a, 1998b) proposed a molar network model, which formally links the observation of widened discriminal distributions to increased levels of internal noise in the older nervous system (cf. Welford, 1981). These authors reported age differences in memory sensitivity in a memory-scanning task (Allen, 1990; Allen, 1991; Allen et al., 1998a) and in a delayed spatial discrimination task (Allen et al., 1998b), and they successfully fit their model to these data (Allen et al., 1998a, 1998b). However, other age-comparative studies using delayed discrimination thresholds (indicative of discriminal dispersion) for size, spatial frequency, or motion (Fahle \& Daum, 1997; Sara \& Faubert, 2000; Faubert \& Bellefeuille, 2002; Bennett, Sekuler, \& Sekuler, 2007) as well as delayed oculomotor (Sweeney, Rosano, Berman, \& Luna, 2001) or manual (Lemay, Bertram, \& Stelmach, 2004) responses have failed to find significant age-related differences in the precision of visual short-term memory (STM). It has been proposed that compensatory reorganization may account for the absence of age-related differences in these studies, in the sense that the negative consequences of deficient sensory processing are attenuated or eliminated by greater reliance on top-down control (Park \& Reuter-Lorenz, 2009). In line with this notion, studies investigating alterations in the neuronal networks at work during delayed matching of sine wave gratings have found an absence of age-related performance differences accompanied by remarkable differences in taskrelevant cortical networks (McIntosh et al., 1999; Bennett, Sekuler, McIntosh, \& Della-Maggiore, 2001; Della-Maggiore et al., 2000). Furthermore, Carp et al. (2010) recently used multivoxel pattern analysis to evaluate the distinctiveness of neuronal activation patterns related to STM in younger and older participants at varying levels of perceptual load. Irrespective of the number of presented items, they found reduced distinctiveness in activation of the early visual cortices of older participants. Agerelated differences in distinctiveness of activation within frontal and parietal lobes, however, depended on the number of presented items. Distinctiveness was comparable over age groups when only one object was presented, but differed remarkably across age groups when more than one object was presented in parallel.

In contrast to the study by Carp et al. (2010), most of the behavioral studies investigating age differences in delayed discrimination have used one item only (McIntosh et al., 1999; Sara \& Faubert, 2000; Della-Maggiore et al., 2000; Bennett et al., 2001). In the light of age-related declines in the distinctiveness of activation patterns, we predict that age-related differences in discrimination performance should be particularly pronounced when more than one item needs to be retained in memory. Research on visual STM in younger adults provides indirect support for this prediction, showing that the precision in free recall for color (Bays, Catalao, \& Husain, 2009; Bays, Wu, \& Husain, 2011; Fougnie, Asplund, \& Marois, 2010; Zhang \& Luck, 2008) and orientation (Anderson, Vogel, \& Awh, 2011), as well as discrimination performance for location information (Bays \& Husain, 2008), vary as a function of the number of presented items. Also, Sara and Faubert (2000) observed age-related increases in discrimination thresholds, which can be seen as indicating increases in discriminal dispersion, in a delayed matching task with multiple targets. Notably, no such age-related differences were present in another reported experiment in which participants only had to discriminate between two consecutively presented items.

To test the prediction that age-related increments in discriminal dispersion come to the fore when more than one item needs to be retained in STM, we adapted a change-detection paradigm for spatial location (Bays \& Husain, 2008; see Figure 1). The paradigm was designed to investigate adult age differences in discriminal dispersion at different levels of perceptual load (one, three, and five items presented simultaneously). At the heart of the task is the delayed discrimination of spatial positions of colored squares, where the spatial position of one of the presented squares has to be evaluated against a subsequently presented test square, which was displaced by one of three different displacement magnitudes either

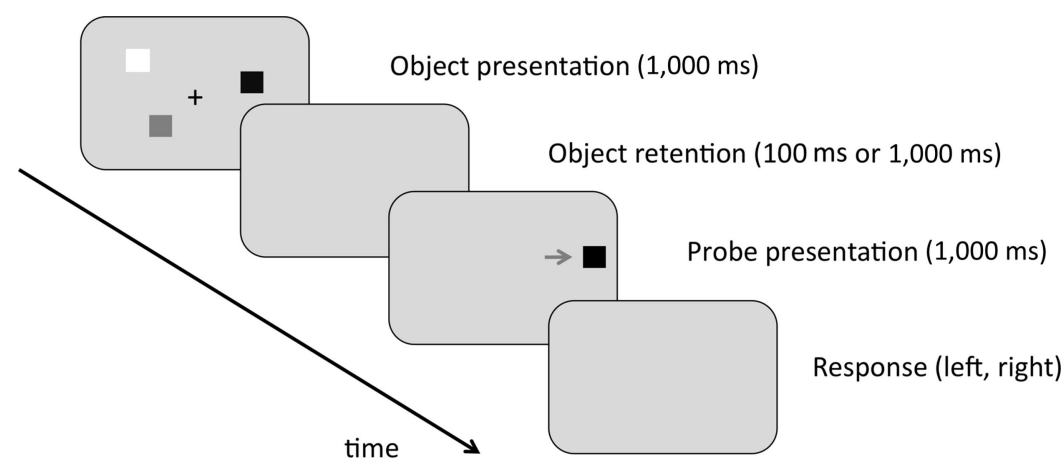

Figure 1. Procedure of the change-detection task. 
to the left or to the right. Assuming that spatial configurations of stimuli presented in the visual environment will correspond to representations on a retinotopic map (Lennie, 1998), we predict that more distant test stimuli will induce more distant and therefore less overlapping representations, which will be detected more readily as being different. According to psychophysics methodology (e.g., Klein, 2001; Kuss, Wichmann, \& Jäckel, 2005b), the functional relationship between stimulus displacement magnitude and discrimination performance can be captured by psychometric functions such as the cumulative normal function characterized by mean and standard deviation. Implicit to this approach is the assumption of graded representations in the sense of bell-shaped discriminal distributions (e.g., Bays \& Husain, 2008; Thurstone, 1929; Wickens, 2002) with intra- and interindividual differences in discriminal dispersions. Based on these assumptions, we may expect that the standard deviation of the psychometric function can inform us about the dispersion of the underlying visuospatial discriminal distribution of any given participant. That is, the standard deviation of the psychometric function is equal to the discriminal dispersion given the simplifying assumption that the probe stimulus is perceived without dispersion.

It is important to note, however, that performance in the task will likely be influenced by another factor, namely, the memory capacity for a given person (Cowan, 2001; Zhang \& Luck, 2008). Capacity refers to the upper limit of integrated item representations that a person can establish and maintain at the same time. If the number of presented items (here, colored squares) exceeds the individual capacity limit, some items will be ignored completely and probes on these items will provoke guessing responses. Because guessing is insensitive to displacement magnitude, discrimination performance will be lowered on all levels of displacement, leading to imperfect discrimination even at the largest possible displacements, that is, at the asymptote. If not properly taken into account, differences in capacity and, thus, in asymptotic discrimination performance may mimic differences in discriminal dispersion (Zhang \& Luck, 2009).

These considerations are particularly critical in the context of our study, because age-related differences in STM capacity have been reported (e.g., Sander, Werkle-Bergner, \& Lindenberger, 2011) so that differences in asymptotic discrimination performance must, therefore, be expected. To take care of this issue, we analyzed performance data with a mixture model approach that allows for separating the effect of discriminal dispersion from the effect of capacity limitations (Bays \& Husain, 2008; Zhang \& Luck, 2008). Furthermore, to gauge the relative contribution of perceptual and memory-related processes to age-related differences in discrimination performance, probes were presented at two different retention intervals: a visual condition (100-ms retention interval) and a memory condition (1,000-ms retention interval).

\section{Methods}

\section{Participants}

A total of 23 younger and 27 older adults were invited to participate in the experiment. To reduce sensory confounds, participants selected for the experiment had to be able to report the direction of a horizontal displacement of one colored square by $3.2^{\circ}$ visual angle correctly in at least 26 of 34 trials (75\% correct).
Based on this criterion, the data of one younger and seven older participants were discarded from the analysis. Thus, the effective sample comprised 22 (13 women; $M_{\text {age }}=25$ years; age range: 20-30) younger and 20 (18 women; $M_{\text {age }}=68$ years; age range: 62-70) older healthy adults. All participants reported normal or corrected-to-normal visual acuity and normal color vision. Computerized testing of visual acuity (Bach, 1996, 2007) at a viewing distance of $2 \mathrm{~m}$ revealed a significant age group difference in visual acuity, $t(40)=4.41, p<.01$; mean decimal visual angle for the young $=1.41$; mean decimal visual angle for the old $=1.06$. A test of color vision (Velhagen, 2003) confirmed perfect color vision for all participants. Compared to their younger counterparts, older participants achieved a lower score on a test of perceptual speed (the Digit Symbol Substitution Test from the Wechsler Adult Intelligence Scale-Revised; Wechsler, 1981), $t(40)=6.88$, $p<.01$; mean performance of the young $=68.6$; mean performance of the old $=47.0$. At the same time, they showed slightly superior performance in a vocabulary test (MehrfachwahlWortschatz-Test, Version A; Lehrl, Merz, Burkhard, \& Fischer, $1991), t(40)=-4.46, p<.01$; mean performance of the young $=$ 31.3; mean performance of the old $=33.6$. Thus, the older adults showed a pattern typical of healthy cognitive aging (cf. Li et al., 2005). All participants gave informed consent to participate prior to testing, and each received $€ 17$ for participating in the experiment. The Ethics Committee of the Max Planck Institute for Human Development approved of the study.

\section{Stimuli}

Experimental stimuli were presented centrally on a LCD display at a viewing distance of $60 \mathrm{~cm}$. A chin rest was used. Each trial started with the presentation of a black central fixation cross on a gray background. After $500 \mathrm{~ms}$, a study display was presented (see Figure 1) showing one, three, or five colored squares $\left(0.8^{\circ} \times 0.8^{\circ}\right.$ visual angle) for $1,000 \mathrm{~ms}$. Item locations were drawn at random locations within an area of $15^{\circ} \times 15^{\circ}$ visual angle around the central fixation cross. Multiple items appearing within one trial were separated by a minimum distance of $3^{\circ}$ visual angle to each other and to the fixation cross. One of five highly distinctive colors (red, green, blue, white, or black) was then randomly assigned to each target positions. After presentation of the study display $(1,000$ $\mathrm{ms}$ ), the display changed to a blank screen for the retention interval of either 100 or 1,000 ms. Finally, the test display consisted of only one probe-square, which was one of the study items shifted either leftward or rightward by $0.8,1.6$, or $3.2^{\circ}$ visual angle. After another $350 \mathrm{~ms}$, the display turned blank again and participants pressed the left or right arrow key on a standard keyboard, indicating the shifting direction.

\section{Design}

The study design consisted of one between-subjects factor (age) and four orthogonally crossed within-subjects factors: set size (one, three, and five squares in the set), target displacement (0.8, 1.6 , and $3.2^{\circ}$ visual angle), displacement direction (left or right), and retention interval (100 and 1,000 ms). Each within-subject factor combination was repeated in 17 trials, resulting in 612 total trials per participant. 


\section{Procedure}

Each session lasted approximately two hours. Testing was conducted in groups of not more than three participants. At the beginning of each session, we assessed visual abilities and processing speed. The experimenter then instructed participants using a slideshow, including one instruction trial. Special emphasis was put on performance accuracy as opposed to speed. Participants started the experiment when the experimenter was certain that they had completely understood the task instructions. The experimental layout and timing was introduced again during 12 practice trials preceding the 612 trials. The participants, once more, were invited to ask questions or to express if anything was unclear. Research assistants attended and supported performance during the practice trials and reinstructed participants whenever they were unable to follow the experimental demands.

Trials followed one after another at a computer-controlled pace. However, participants were free to take a break after blocks of twenty trials lasting about one to two minutes. Two major breaks were included. During these breaks, the experimenter turned off the computer displays and participants were asked to fill in a questionnaire or do the vocabulary test, which was an untimed paper-and-pencil test.

\section{Apparatus}

The experiment was conducted on a $2.8 \mathrm{GHz}$ Intel Core2Duo Quad Dell PC (Dell Inc.) presenting stimuli on a 19-in. Eizo FlexScan S1901 LCD screen (Eizo Nanao Corp.). Participants responded on a standard personal computer keyboard. Experimental display and response collection was implemented in Matlab (The MathWorks Inc.) code, using the Psychophysics Toolbox (Brainard, 1997; Pelli, 1997).

\section{Data Analysis}

We obtained binary data from every trial indicating whether the participant judged the item displacement correctly (e.g., indicating to the right when displacement was actually to the right) or not. The model described below was fit to the binomial data obtained for each participant at each retention interval and each set size.

\section{Modeling}

As described above, we assumed that delayed discrimination performance at a given displacement magnitude would be determined by two sources: (a) STM capacity (i.e., the probability to encode and maintain an item) and (b) the shape of the psychometric function linking displacement magnitude to discrimination performance. Under conditions of suprathreshold stimuli, delayed discrimination performance, $p_{\text {correct }}$, would be fully determined by the memory capacity, $k$, the number of presented items, $n_{\text {item }}$, and the number of available response options, $n_{\text {opt }}$, determining the guessing rate $g=1 / n_{\mathrm{opt}}$. If more items are presented than representations can be formed, $n_{\text {item }}>k$, then the probability of responding correctly is determined by $p_{\text {correct }}=k / n_{\text {item }}+(1-$ $\left.k / n_{\text {item }}\right) * g$, otherwise $p_{\text {correct }}=1$. In the present study, however, expected delayed discrimination performance, $p_{\text {correct, }}$ was not equal to 1 if an item was in memory, because probe stimuli were presented such that displacements, $d$, were close to the detection threshold for a given person. Discrimination performance, thus, also depended on the magnitude of the probe displacements, $d$. Specifically, the nonlinear relationship between the displacement magnitude and the probability of responding "to the right" was modeled with a psychometric function, which was a Gaussian distribution function, $\Phi$, with the mean, $\mu$, and the standard deviation, $\sigma$, such that $p_{\text {right }}=\Phi(d, \mu, \sigma)$.

Taking the two sources together, the probability of responding "to the right," $F$, as given by the mixture model, is:

$$
F(d, \pi, \sigma, \mu)=\pi * \Phi[(d-\mu) / \sigma]+(1-\pi) * g,
$$

where the mixture parameter, $\pi=k / n_{\text {item }}$, reflects the probability that an item is in memory (i.e., the effect of STM capacity), $g=$ $1 / 2$ is the guessing rate or the probability of choosing "right" by chance, $\mu$ is the bias parameter, which has been set to 0 here, ${ }^{2}$ and $\sigma$ is the standard deviation, which represents discriminal dispersion.

We estimated the parameters using Bayesian inference (Sivia, 2004), following the Markov Chain Monte Carlo procedures described by Kuss, Jäkel, and Wichmann (2005a, 2005b; see Appendix). In Bayesian inference, a posterior probability distribution of the parameters given the data is created from the likelihood of the parameters given the data and the prior knowledge on the parameters. Numerical methods are used to sample from this posterior probability distribution, and point estimates of the parameters were obtained by taking the median of the marginal posterior distributions for the single parameters (i.e., $\sigma$ and $\pi$ in the present case).

All modeling was implemented in the $\mathrm{R}$ environment for statistical computing (R-core) using an adapted version of the PsychoFun package provided by Kuss, Jäkel, and Wichmann (2005b). The resulting parameter estimates were analyzed using repeatedmeasures multivariate analysis of variance (MANOVA) because violations of the sphericity assumption were likely to occur in the present design. MANOVA can be applied under the assumption of an unstructured covariance matrix (Maxwell \& Delaney, 2004). We used Wilks's lambda to test for statistical significance, and $t$-tests for follow-up analyses of statistically significant effects. The level of significance was set to $\alpha=.05$, except for post hoc comparisons for which we chose $\alpha=.01$ to control for alpha-error accumulation. We report partial eta squared as a measure of effect size.

\section{Results}

Data were screened a priori with respect to reaction times. Because we strongly emphasized accuracy and some older participants often visually checked whether they were pressing the correct buttons, we chose a liberal fixed cutoff value of $4 \mathrm{~s}$ for excluding responses. Less than $1 \%$ of all trials had to be discarded due to this criterion. The greatest data loss at the individual level due to this criterion amounted to $3.8 \%$ of the trials.

Our main measures of interest were the dispersion, $\sigma$, of a cumulated normal distribution, indicative of discriminal disper-

\footnotetext{
${ }^{2}$ We tested the effect of set size, retention time, or age on response bias fitting a three-parameter model to the data where the central tendency measure, $\mu$, was free. We did not find significant effects of set size, retention time, or age on $\mu$.
} 
sion, and the mixture parameter, $\pi$, which reflects asymptotic discrimination performance by giving the probability of an item being in memory. Adequacy of the resulting mixture model was first investigated by fitting the model to accuracy data aggregated over participants within each age group, each set size, and each condition. At the group level, the model fit the data very well, with no less than $99 \%$ of the variance accounted for in any of the conditions. Second, we investigated the adequacy of the model separately in each participant for every condition. Individual fits were generally acceptable (see Figure 2) with slightly inferior fits among older adults, especially at set size five and for the longer retention time condition. ${ }^{3}$

\section{Dispersion Parameter}

Descriptive statistics are reported in Table 1. Younger participants generally showed lower dispersion of the psychometric function than older adults, $F(1,40)=28.84, p<.01, \eta^{2}=.42$, and dispersion was higher at longer than at shorter retention intervals, $F(1,40)=130,49, p<.01, \eta^{2}=.77$. The interaction between age and retention interval (see Figure 3 ) was not reliable, $F(1,40)=2.79, p>.05, \eta^{2}=.07$. In other words, age-related differences in dispersion observed at the retention interval of 100 ms did not increase when the retention interval was extended to $1,000 \mathrm{~ms}$.

The load manipulation was effective in changing the dispersion of the psychometric function, $F(2,39)=143.66, p<.01, \eta^{2}=$ .88. The linear contrast was highly significant, $F(1,40)=212.39$, $p<.01, \eta^{2}=.84$. Furthermore, post hoc analyses revealed a significant difference between set size one and set size three, $t(41)=-10.14, p<.01$, but also a significant difference between set size three and set size five, $t(41)=-3.78, p<.01$. However, the reliable quadratic contrast, $F(1,40)=14.58, p<.01, \eta^{2}=$ .27 , and an inspection of Figure 4 indicate that the increase was more pronounced from set size one to set size three than it was from set size three to set size five, $t(41)=3.61, p<.01$.

Of note, and as predicted, we observed a significant age by set size interaction for dispersion, $F(2,39)=17.35, p<.01, \eta^{2}=$ .47. At set size one, the two age groups exhibited the same level of dispersion, $t(40)=-1.36, p>.01$. However, they strongly differed from each other at set size three, $t(40)=-4.73, p<.01$. Younger and older adults did not differ reliably in the dispersion increase from set size three to set size five, $t(40)=-0.69, p=$ .493. Thus, the observed interaction between age group and set size was primarily due to age differences in dispersion increase from set size one to set size three. Both age groups showed reliable differences between the two retention time conditions at set size one, young: $t(21)=-4.31, p<.01$; old: $t(21)=-5.16, p<.01$, suggesting that they were not performing at ceiling, at least not in the longer retention time condition. Retention time did not reliably modulate the effects of age group and set size, $F(2,39)<1$ (see Figure 4).

\section{Mixture Parameter}

The mixture parameter, $\pi$, represented asymptotic discrimination performance (i.e., the probability that an item was held in memory). Descriptive statistics for the estimates of this parameter are reported in Table 1 . The two age groups differed significantly in terms of $\pi, F(1,40)=8.32, p<.01, \eta^{2}=.17$, indicating that older participants built up or maintained fewer items in memory than younger participants. Moreover, $\pi$ decreased with increasing retention time, $F(1,40)=17.34, p<.01, \eta^{2}=.30$. This decrease, however, did not reliably interact with age group, $F(1,40)<1$ (see Figure 3).

If $\pi$ was related to the memory capacity, $k$, in the sense of indicating the maximum number of integrated item representations, then we must expect a decrease with increasing load, $n$, because $\pi=k / n$. The reliable main effect of load, $F(2,39)=$ 93.66, $p<.01, \eta^{2}=.83$, confirmed this expectation. A strong linear contrast, $F(1,40)=185.01, p<.01, \eta^{2}=.82$, together with the absence of a reliable quadratic contrast, $F(1,40)=2.32, p>$ $.05, \eta^{2}=.06$, suggested that this decrease was primarily linear in nature. The decrease in $\pi$ from set size one to set size three did not differ from the decrease from set size three to set size five, $t(41)=$ $1.52, p>.01$, further supporting the notion of linearity.

The effect of load on the mixture parameter, $\pi$, was particularly pronounced in older participants, as indicated by a significant age by set size interaction, $F(2,39)=5.03, p<.05, \eta^{2}=.21$. Together with the linear contrast, $F(1,40)=9.95, p<.01, \eta^{2}=$ .20 , this result is in line with the assumption that older participants represented fewer items in memory than their younger counterparts. Though Figure 4 suggests the presence of an age-related difference in $\pi$ at set size one, this impression was not statistically reliable at either retention interval, short: $t(40)=1.30, p>.01$; long: $t(40)=1.87, p>.01$; combined: $t(40)=1.95, p>.01$. Similarly, though numerically increasing in magnitude, the agerelated difference in $\pi$ was not reliable at set size three, $t(40)=$ $2.19, p>.01$. Age group differences were reliable, $t(40)=3.21$, $p<.01$, however, at set size five. Finally, the triple interaction between age group, retention interval, and set size was not reliable, $F(2,37)<1$. In sum, age group differences in $\pi$ were already present at very short retention intervals and did not differ reliably between the two retention time conditions.

\section{Discussion}

\section{Rationale and Results}

Our study was guided by the neural noise hypothesis of aging (Welford, 1981, 1984) as well as related predictions of greater discriminal dispersion (Allen et al., 1998a, 1998b), and reduced distinctiveness of neural representations (Li et al., 2000; Li \& Sikström, 2002) with advancing adult age. Evidence from animal models (Hua et al., 2006; Liang et al., 2010; Yang et al., 2009) has suggested higher levels of neural noise in the aging nervous system. In addition, age-comparative functional neuroimaging studies in humans have supported the prediction that neural distinctiveness decreases from early to late adulthood (Carp et al., 2010; Carp et al., 2011; Goh et al., 2010; Park et al., 2004; Park et al., 2010; Payer et al., 2006; Voss et al., 2008). At the same time,

\footnotetext{
${ }^{3}$ Individual model fits were not consistently good, with explained variance of less than $70 \%$ in some cases (2\% of all cases in the young and $7.5 \%$ of all cases in the old). To see whether bad fit caused any bias in terms of mean differences, we reran the analysis excluding those cases where $R^{2}<$ 0.7. The pattern of results was identical to those reported.
} 

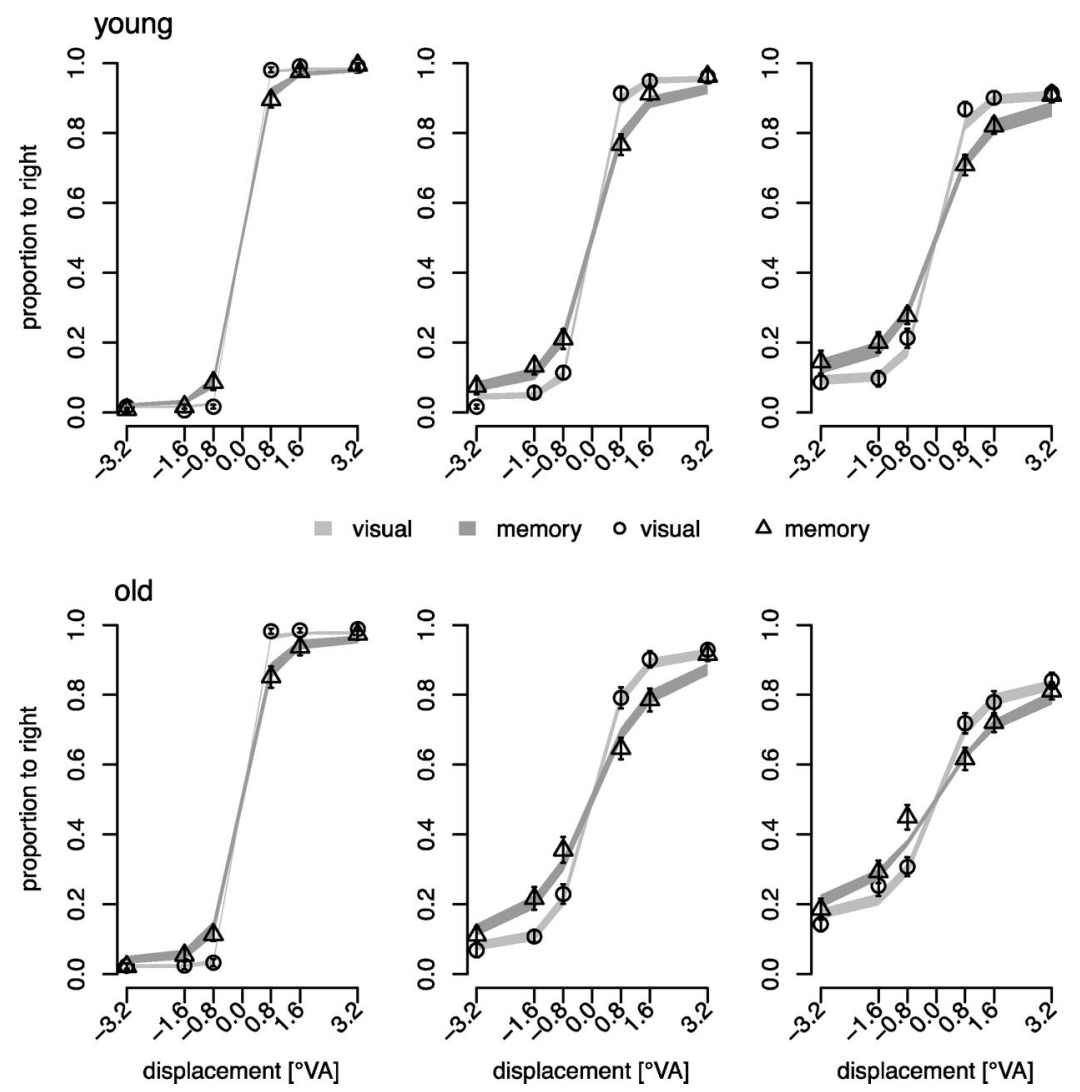

Figure 2. The goodness of the model fit can be seen in the younger (upper row) and older (lower row) participants. Data points reflect the mean accuracy with standard errors. The shaded areas show the mean of the individual model fits surrounded by one standard error. Note the high degree of overlap between the $68 \%$ confidence intervals for accuracy data and the corresponding model fits. VA $=$ visual angle

demonstrations of altered discriminal dispersion in humans have been sparse (but see Allen et al., 1998a, 1998b; Sara \& Faubert, 2000). Informed by findings of increasing discriminal dispersion with greater STM load in younger adults (Anderson et al., 2011; Bays et al., 2009; Bays \& Husain, 2008; Bays, Wu, \& Husain, 2011; Fougnie et al., 2010; Zhang \& Luck, 2008), we hypothesized that increasing set size would exacerbate age-related differences in discriminal dispersion, which we conceptualized as a behavioral indicator of the dispersion of probabilistic perceptual and memory representations.
We addressed this hypothesis using a change-detection paradigm for visuospatial location (Bays \& Husain, 2008) with three different set sizes at two different retention times. Test items were presented at one of six different displacement magnitudes. Discrimination performance was modeled with a mixture model (Zhang \& Luck, 2008) that captures the relationship between displacement magnitude and discrimination performance as a scaled cumulative Gaussian distribution. The standard deviation, $\sigma$, of this psychometric function reflects the change of performance with displacement magnitude, and the mixture parameter,

Table 1

Means and Standard Deviations of Discriminal Dispersion $(\sigma)$ and Mixture Parameter $(\pi)$ Estimates

\begin{tabular}{|c|c|c|c|c|c|c|c|c|c|}
\hline & & \multicolumn{4}{|c|}{ Younger (20-30 years) } & \multicolumn{4}{|c|}{ Older (60-70 years) } \\
\hline \multicolumn{2}{|c|}{ Measure } & \multicolumn{2}{|c|}{$\sigma$} & \multicolumn{2}{|c|}{$\pi$} & \multicolumn{2}{|c|}{$\sigma$} & \multicolumn{2}{|c|}{$\pi$} \\
\hline Retention & Load & $M$ & $S D$ & $M$ & $S D$ & $M$ & $S D$ & $M$ & $S D$ \\
\hline \multirow[t]{3}{*}{$100 \mathrm{~ms}$} & 1 & 0.32 & 0.06 & 0.97 & 0.03 & 0.33 & 0.09 & 0.95 & 0.04 \\
\hline & 3 & 0.53 & 0.17 & 0.91 & 0.08 & 0.84 & 0.32 & 0.85 & 0.11 \\
\hline & 5 & 0.64 & 0.20 & 0.81 & 0.13 & 1.09 & 0.54 & 0.77 & 0.12 \\
\hline \multirow{3}{*}{$1,000 \mathrm{~ms}$} & 1 & 0.53 & 0.23 & 0.96 & 0.04 & 0.63 & 0.28 & 0.92 & 0.09 \\
\hline & 3 & 0.90 & 0.35 & 0.86 & 0.13 & 1.41 & 0.49 & 0.78 & 0.15 \\
\hline & 5 & 1.09 & 0.42 & 0.75 & 0.19 & 1.60 & 0.45 & 0.62 & 0.18 \\
\hline
\end{tabular}



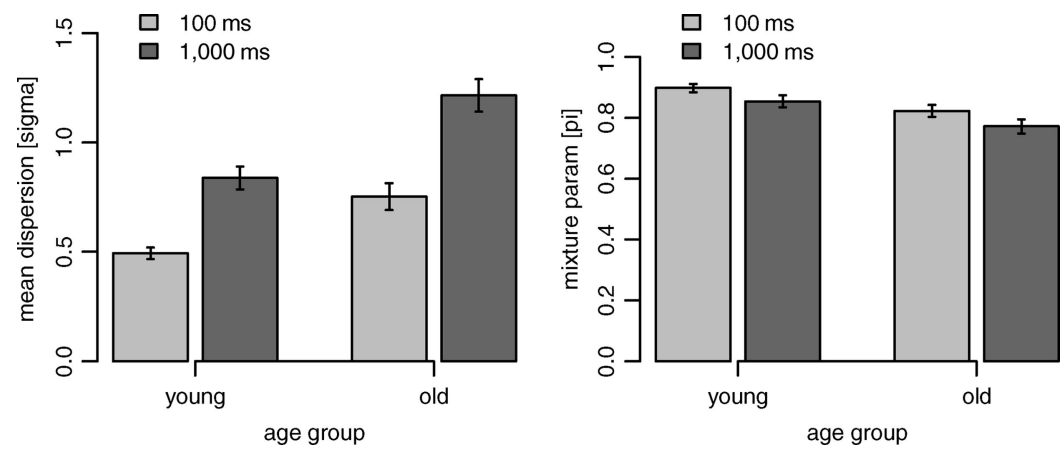

Figure 3. The main effect of age and of condition for both discriminal dispersion (left) and probability of an item to be represented in memory (right). There were no interactions between age group and retention time condition for either measure.

$\pi$, determines the deviation from perfect performance at the asymptote. According to earlier work (Bays \& Husain, 2008; Zhang \& Luck, 2008), we interpreted these parameters in the sense of discriminal dispersion and the probability to encode and maintain an item (i.e., the effect of STM capacity), respectively.

In line with our predictions, we observed age-related differences in discriminal dispersion when set sizes were greater than 1 . Older participants were as able as younger adults to detect changes in item location at a set size of 1 , but were less able than younger adults to discriminate location differences when multiple items were presented. In addition, we found age-related differences in asymptotic discrimination performance, which increased with increasing set size, pointing to an age-related decrease in STM capacity. In sum, the older adults of the present study represented a lower number of items at a lower resolution than younger adults when faced with set sizes greater than 1 . These adult age differences were not reliably modulated by retention interval.

\section{Study Limitations}

The high degree of selectivity of the sample of older adults participating in this study limits the generalizability of our results to the population of aging individuals. About one third of the older participants were excluded from data analysis because perfor- mance with only one item and the largest displacement was too low. Because the corresponding changes were far above the detection threshold for all participants, low performance may indicate difficulties in understanding the task that went unnoticed during the instruction phase of the experiment. However, the selective exclusion of older adults with low levels of performance reduced our chances of observing age-related decline.

\section{Comparison to Earlier Studies}

Our results resemble previous findings on age-related differences in discrimination performance in two ways. First, in agreement with various studies investigating discrimination performance in simple visual or visuospatial STM tasks (Bennett et al., 2001; Bennett et al., 2007; Della-Maggiore et al., 2000; Fahle \& Daum, 1997; Faubert \& Bellefeuille, 2002; Lemay et al., 2004; McIntosh et al., 1999; Sara \& Faubert, 2000; Sweeney et al., 2001), age-related differences in discriminal dispersion were not reliable at set size one. This result is unlikely to be entirely due to ceiling because effects of retention interval were present in both age groups. Second, in line with our hypothesis and in line with data obtained by Sara and Faubert (2000), age differences in discrimination performance surfaced when task difficulty increased and participants had to process more than one item in
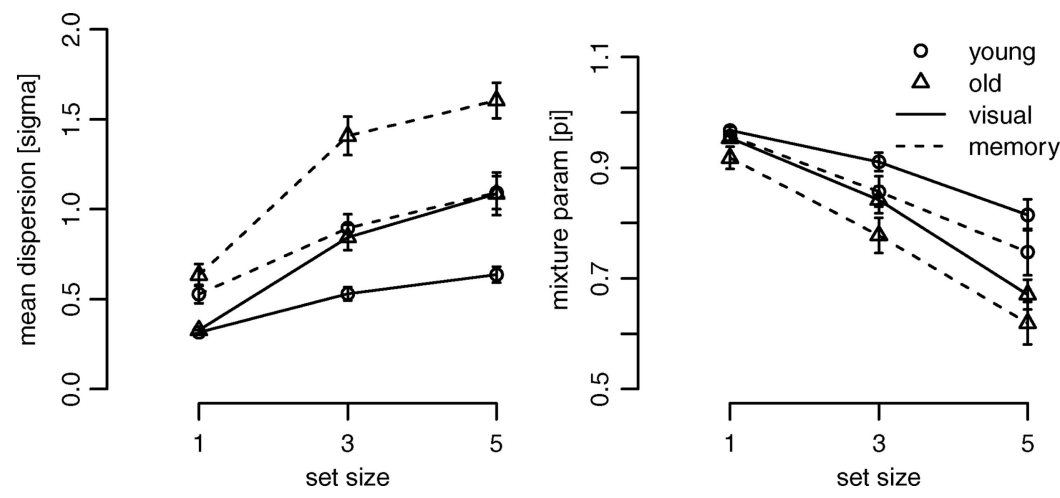

Figure 4. A strong modulation of discriminal dispersion (left) and probability of an item to be represented in memory (right) by set size was present in younger and older participants. In discriminal dispersion, a decelerated increase from set size three to set size five is visible at both retention time conditions and for both age groups. 
parallel (see also Allen et al., 1998b). The origins of this simultaneous access deficit are unclear. Faubert and colleagues (2002; Sara \& Faubert, 2000) have proposed that higher processing resource requirements might emerge in the older brain due to noisier sensory signals (Hua et al., 2006; Liang et al., 2010; Yang et al., 2009). If attentional resources are limited, performance differences may surface at higher levels of task difficulty, when greater effort is no longer sufficient to "compensate," or attenuate, the repercussions of imprecise percepts (Carp et al., 2010; Reuter-Lorenz \& Cappell, 2008; Schneider-Garces et al., 2010). Thus, larger age differences in discriminal dispersion with greater set size may represent the interaction of multiple sources, such as reduced signal-to-noise ratio in early visual processing and the ability to cope with them through top-down modulation (Reynolds \& Chellazi, 2004). The exact contribution of each of these possible mechanisms, however, cannot be separated from each other based on the present data.

To separate age-related differences in perceptual processing from differences in memory-related processing, we tested two retention intervals, 100 and $1,000 \mathrm{~ms}^{4}$ In agreement with earlier studies using long retention intervals (up to $10 \mathrm{~s}$; Bennett et al., 2007; Della-Maggiore et al., 2000; Fahle \& Daum, 1997; Lemay et al., 2004; McIntosh et al., 1999; Sara \& Faubert, 2000; Sweeney et al., 2001) or masks (Bennett et al., 2001; Faubert \& Bellefeuille, 2002) in delayed discrimination tasks, the effect of retention time on discriminal dispersion did not differ reliably across age groups. Note, however, that our longer retention interval was rather short and might still have allowed, to some extent, for contributions from iconic memory (Phillips, 1974). Conversely, studies using longer retention intervals, complex stimuli, or a combination thereof may lead to different results. For example, Allen et al. (1998a) detected a reliable interaction between retention time and age for discriminal dispersion using the Sternberg task to probe primary (100-ms masked retention) and secondary memory (10-s retention with distracter task). This task, more than delayed discrimination, may have posed greater demands on associative binding, which is particularly susceptible to the adverse effects of aging (e.g., Chalfonte \& Mitchell, 1996; Li, Naveh-Benjamin, \& Lindenberger, 2005; Naveh-Benjamin, 2000; Old \& Naveh-Benjamin, 2008; Shing et al., 2010).

Our analysis was based on a mixture model (Zhang \& Luck, 2008) separating the effect of discriminal dispersion for spatial location from the effect of STM capacity (Cowan, 2001), represented by asymptotic discrimination performance. In line with earlier reports on age-related differences in memory capacity (Cowan, Naveh-Benjamin, Kilb, \& Saults, 2006; Sander et al., 2011), we found age differences in asymptotic discrimination performance. Specifically, older adults were less likely to hold items in memory than younger participants. According to the mixture model, limits on STM capacity are a stable individual characteristic and should remain unaffected by load, which was operationalized as set size in the present study. The effect of this limitation on asymptotic discrimination performance, however, should increase with increasing load. Thus, the reliable interaction between age and set size for this measure further supports the claim that younger and older participants differ in STM capacity. In contrast, longer retention time decreased asymptotic discrimination performance in both age groups but did not exacerbate age differences, again pointing to the importance of early perceptual processes for explaining the age differences observed in this study.

Based on these results, we conclude that visuospatial representations undergo both gradual degradation as well as abrupt decay over the first second of retention. This conclusion is somewhat inconsistent with the results of Zhang and Luck (2009), who found that longer retention intervals affected the probability of an item to be in memory, but not discriminal dispersion. Zhang and Luck (2009) investigated more retention intervals between 1 and $10 \mathrm{~s}$, which might account for this discrepancy in results. Future studies should include retention times in the range from $100 \mathrm{~ms}$ to $10 \mathrm{~s}$ to investigate this issue more closely.

\section{Models of Short-Term Memory Capacity}

The interpretation of the present results is contingent on the model of capacity limitations in visual STM that is used. Here, we preferred a rather general mixture model with separate parameters for discriminal dispersion and the probability of an item to be in memory to simultaneously estimate age-related differences in these two aspects of the task. This model posits inter- and intraindividual variation in discriminal dispersion and interindividual variation in STM capacity. At least two recent models on performance limits in visual STM do not share this premise.

The slots model proposed by Zhang and Luck (2008) is based on the assumption of an object-based capacity limit, with no intraindividual differences in discriminal dispersion (e.g., in response to variations in set size) for items that the participant is able to represent (see also Cowan, 2001; Luck \& Vogel, 1997; Rouder et al., 2008). In contrast, the continuous resource model advocated by Bays and Husain (2008; see also Bays et al., 2009) assumes that representations are not lost completely. According to their model, all observed differences in memory performance can be traced back to differences in discriminal dispersion. On a qualitative level of description, the present data are not entirely consistent with the predictions of either of model. First, we found an increase in discriminal dispersion from set size one to set size three, which is at odds with the assumption of load-independent discriminal distributions of the slots model (at least in its pure form). Second, the monotonic decrease in asymptotic discrimination performance speaks against the continuous resource model, which predicts perfect asymptotic discrimination performance irrespective of set size. Though in partial disagreement with either model, the observed pattern of decreasing probability of an item being in memory and increasing discriminal dispersion is consistent with the empirical results of earlier studies (Anderson et al., 2011; Bays et al., 2009; Fougnie et al., 2010; Zhang \& Luck, 2008).

Recently, a third framework, neural object-file theory (Xu \& Chun, 2009), has been introduced, which combines features of the slots model and the continuous resource model. Though not math-

\footnotetext{
${ }^{4}$ We also tested whether age-related differences in visual acuity would statistically account for the observed age effects on discriminal dispersion. Note that our participants were screened for visual ability and that both age groups performed at comparable levels at set size one, which suggests that all participants were generally able to detect changes of the magnitude tested here. In a follow-up analysis, we controlled statistically for visual acuity and found a similar pattern of effects of age, set size, and retention time on discriminal dispersion.
} 
ematically formalized, this framework lends a viable explanation of the present pattern of findings (Anderson et al, 2011) based on findings from neuroimaging studies. Specifically, $\mathrm{Xu}$ and Chun (2006, 2009) distinguish between attentional capacity (i.e., the number of items that can be attended or represented) and the fidelity of low-level sensory processing. According to their framework, attentional foci act on integrated representations and may be limited in number. Precision of these integrated representations is affected by sensory processing fidelity, which may depend on the amount and the complexity of the stimulus material (Alvarez \& Cavanagh, 2004; Eng, Chen, \& Jiang, 2005; Xu \& Chun, 2006) as well as properties of the sensory system (Scalf \& Beck, 2010). The theory allows for increasing discriminal dispersion with greater set size (Miller, Gochin, \& Gross, 1993; Beck \& Kastner, 2005; Scalf \& Beck, 2010; Xu \& Chun, 2006), and for between-person differences in STM capacity, or asymptotic discrimination performance (Cowan, 2001; Todd \& Marois, 2004). Our data suggest that both constraints are present, and that both increase with advancing adult age

\section{References}

Allen, P. A. (1990). Influence of processing variability on adult age differences in memory distribution of order information. Cognitive Development, 5, 177-192. doi:10.1016/0885-2014(90)90025-O

Allen, P. A. (1991). On age differences in processing variability and scanning speed. Journals of Gerontology, 46, 191-201. doi:10.1093/ geronj/46.5.P191

Allen, P. A., Kaufman, M., Smith, A. F., \& Propper, R. E. (1998a). Age differences in entropy: Primary versus secondary memory. Experimental Aging Research, 24, 307-336. doi:10.1080/036107398244175

Allen, P. A., Kaufman, M., Smith, A. F., \& Propper, R. E. (1998b). A molar entropy model of age differences in spatial memory. Psychology and Aging, 13, 501-518. doi:10.1037/0882-7974.13.3.501

Alvarez, G. A., \& Cavanagh, P. (2004). The capacity of visual short-term memory is set both by visual information load and by number of objects. Psychological Science, 15, 106-111. doi:10.1111/j.0963-7214 .2004.01502006.x

Anderson, D. E., Vogel, E. K., \& Awh, E. (2011). Precision in working memory reaches a stable plateau when individual item limits are exceeded. The Journal of Neuroscience, 31, 1128-1138. doi:10.1523/ JNEUROSCI.4125-10.2011

Bach, M. (1996). The Freiburg Visual Acuity test-automatic measurement of visual acuity. Optometry and Vision Science, 73, 49-53. doi: 10.1097/00006324-199601000-00008

Bach, M. (2007). The Freiburg Visual Acuity Test-variability unchanged by post-hoc re-analysis. Graefe's Archive for Clinical and Experimental Ophthalmology, 245, 965-971. doi:10.1007/s00417-006-0474-4

Balota, D. A., Dolan, P. O., \& Duchek, J. M. (2000). Memory changes in healthy older adults. In E. Tulving \& F. I. M. Craik (Eds.), The Oxford handbook of memory (pp. 395-409). New York, NY: Oxford University Press.

Bays, P. M., Catalao, R. F., \& Husain, M. (2009). The precision of visual working memory is set by allocation of a shared resource. Journal of Vision, 9, 1-11. doi:10.1167/9.10.7

Bays, P. M., Wu, E. Y., \& Husain, M. (2011). Storage and binding of object features in visual working memory. Neuropsychologia, 49, 16221631. doi:10.1016/j.neuropsychologia.2010.12.023

Bays, P. M., \& Husain, M. (2008). Dynamic shifts of limited working memory resources in human vision. Science, 321, 851-854. doi: $10.1126 /$ science. 1158023

Beck, D., \& Kastner, S. (2005). Stimulus context modulates competition in human extrastriate cortex. Nature Neuroscience, 8, 1110-1116. doi $10.1038 / \mathrm{nn} 1501$

Bennett, P. J., Sekuler, A. B., McIntosh, A. R., \& Della-Maggiore, V. (2001). The effects of aging on visual memory: Evidence for functional reorganization of cortical networks. Acta Psychologica, 107, 249-273. doi:10.1016/S0001-6918(01)00037-3

Bennett, P. J., Sekuler, R., \& Sekuler, A. B. (2007). The effects of aging on motion detection and direction identification. Vision Research, 47, 799-809. doi:10.1016/j.visres.2007.01.001

Brainard, D. H. (1997). The Psychophysics Toolbox. Spatial Vision, 10, 433-436. doi:10.1163/156856897X00357

Braver, T. S., \& Barch, D. M. (2002). A theory of cognitive control, aging cognition, and neuromodulation. Neuroscience and Biobehavioral Reviews, 26, 809-817. doi:10.1016/s0149-7634(02)00067-2

Carp, J., Gmeindl, L., \& Reuter-Lorenz, P. A. (2010). Age differences in the neural representation of working memory revealed by multi-voxel pattern analysis. Frontiers in Human Neuroscience, 4, 217. doi:10.3389/ fnhum.2010.00217

Carp, J., Park, J., Polk, T. A., \& Park, D. C. (2011). Age differences in neural distinctiveness revealed by multi-voxel pattern analysis. Neuroimage, 56, 736-743. doi:10.1016/j.neuroimage.2010.04.267

Chalfonte, B. L., \& Johnson, M. K. (1996). Feature memory and binding in young and older adults. Memory \& Cognition, 24, 403-416. doi: 10.3758/BF03200930

Cowan, N. (2001). The magical number 4 in short-term memory: A reconsideration of mental storage capacity. Behavioral and Brain Sciences, 24, 87-114. doi:10.1017/S0140525X01003922

Cowan, N., Naveh-Benjamin, M., Kilb, A., \& Saults, J. S. (2006). Lifespan development of visual working memory: When is feature binding difficult? Developmental Psychology, 42, 1089-1102. doi:10.1037/ 0012-1649.42.6.1089

Della-Maggiore, V., Sekuler, A., Grady, C., Bennett, P., Sekuler, R., \& McIntosh, A. (2000). Corticolimbic interactions associated with performance on a short-term memory task are modified by age. Journal of Neuroscience, 20, 8410-8416. doi:20/22/8410

Eng, H. Y., Chen, D., \& Jiang, Y. (2005). Visual working memory for simple and complex visual stimuli. Psychonomic Bulletin \& Review, 12, 1127-1133. doi:10.3758/BF03206454

Fahle, M., \& Daum, I. (1997). Visual learning and memory as functions of age. Neuropsychologia, 35, 1583-1589. doi:10.1016/S00283932(97)00069-9

Faubert, J. (2002). Visual perception and aging. Canadian Journal of Experimental Psychology/Revue Canadienne de Psychologie Expérimentale, 56, 164-176. doi:10.1037/h0087394

Faubert, J., \& Bellefeuille, A. (2002). Aging effects on intra- and interattribute spatial frequency information for luminance, color, and working memory. Vision Research, 42, 369-378. doi:10.1016/S00426989(01)00292-9

Fougnie, D., Asplund, C. L., \& Marois, R. (2010). What are the units of storage in visual working memory? Journal of Vision, 10, 27. doi $10.1167 / 10.12 .27$

Goh, J. O., Suzuki, A., \& Park, D. C. (2010). Reduced neural selectivity increases fMRI adaptation with age during face discrimination. Neuroimage, 51, 336-344. doi:10.1016/j.neuroimage.2010.01.107

Grady, C. L., \& Craik, F. (2000). Changes in memory processing with age. Current Opinion in Neurobiology, 10, 224-231. doi:10.1016/S09594388(00)00073-8

Hoyer, W. J., \& Verhaeghen, P. (2006). Memory aging. In J. E. Birren, K. W. Schaie, \& R. P. Abeles (Eds.), Handbook of the psychology of aging (Vol. 6, pp. 209-232). Amsterdam, Netherlands: Elsevier. doi: 10.1016/B978-012101264-9/50013-6

Hua, T., Li, X., He, L., Zhou, Y., Wang, Y., \& Leventhal, A. (2006). Functional degradation of visual cortical cells in old cats. Neurobiology of Aging, 27, 155-162. doi:10.1016/j.neurobiolaging.2004.11.012 
Klein, S. A. (2001). Measuring, estimating, and understanding the psychometric function: A commentary. Perception and Psychophysics, 63, 1421-1455. doi:10.3758/bf03194552

Kuss, M., Jäkel, F., \& Wichmann, F. A. (2005a). Approximate Bayesian inference for psychometric functions using MCMC sampling (Technical Report No. 135). Tübingen, Germany: Max Planck Institute for Biological Cybernetics.

Kuss, M., Jäkel, F., \& Wichmann, F. A. (2005b). Bayesian inference for psychometric functions. Journal of Vision, 5, 478-492. doi:10:1167/ 5.5 .8

Lehrl, S., Merz, J., Burkhard, G., \& Fischer, S. (1991). MehrfachwahlWortschatz-Intelligenztest, MWT-A. Göttingen, Germany: Hogrefe.

Lemay, M., Bertram, C., \& Stelmach, G. (2004). Pointing to an allocentric and egocentric remembered target in younger and older adults. Experimental Aging Research, 30, 391-406. doi:10.1080/03610730490484443

Lennie, P. (1998). Single units and visual cortical organization. Perception, 27, 889-935. doi:10.1016/S0959-4388(00)00073-8

Li, S.-C., Lindenberger, U., \& Frensch, P. A. (2000). Unifying cognitive aging: From neuromodulation to representation to cognition. Neurocomputing: An International Journal, 32-33, 879-890. doi:10.1016/S09252312(00)00256-3

Li, S.-C., Lindenberger, U., \& Sikström, S. (2001). Aging cognition: From neuromodulation to representation. Trends in Cognitive Sciences, 5, 479-486. doi:10.1016/S1364-6613(00)01769-1

Li, S.-C., Naveh-Benjamin, M., \& Lindenberger, U. (2005). Aging neuromodulation impairs associative binding: A neurocomputational account. Psychological Science, 16, 445-450. doi:10.1111/j.0956-7976.2005 .01555.x

Li, S.-C., \& Sikström, S. (2002). Integrative neurocomputational perspectives on cognitive aging, neuromodulation, and representation. Neuroscience and Biobehavioral Reviews, 26, 795-808. doi:10.1016/s0149-7634(02)00066-0

Li, S.-C., Lindenberger, U., Hommel, B., Aschersleben, G., Prinz, W., \& Baltes, P. B. (2004). Transformations in the couplings among intellectual abilities and constituent cognitive processes across the life span. Psychological Science, 15, 155-163. doi:10.1111/j.0956-7976.2004 .01503003.x

Liang, Z., Yang, Y., Li, G., Zhang, J., Wang, Y., Zhou, Y., \& Leventhal, A. G. (2010). Aging affects the direction selectivity of MT cells in rhesus monkeys. Neurobiology of Aging, 31, 863-873. doi:10.1016/ j.neurobiolaging.2008.06.013

Light, L. L. (1991). Memory and aging: Four hypotheses in search of data. Annual Review of Psychology, 42, 333-376. doi:10.1146/annurev.ps 42.020191.002001

Luck, S. J., \& Vogel, E. K. (1997). The capacity of visual working memory for features and conjunctions. Nature, 390, 279-281. doi:10.1038/36846

Maxwell, S. E., \& Delaney, H. D. (2004). Designing experiments and analyzing data: A model comparison perspective (2nd ed.). Mahwah, NJ: Erlbaum.

McIntosh, A. R., Sekuler, A. B., Penpeci, C., Rajah, M. N., Grady, C. L., Sekuler, R., \& Bennett, P. J. (1999). Recruitment of unique neural systems to support visual memory in normal aging. Current Biology, 9, 1275-1278. doi:10.1016/S0960-9822(99)80512-0

Miller, E., Gochin, P., \& Gross, C. (1993). Suppression of visual responses of neurons in inferior temporal cortex of the awake macaque by addition of a second stimulus. Brain Research, 616, 25-29. doi:10.1016/00068993(93)90187-R

Naveh-Benjamin, M. (2000). Adult age differences in memory performance: Tests of an associative deficit hypothesis. Journal of Experimental Psychology: Learning, Memory, and Cognition, 26, 1170-1187. doi:10.1037//0278-7393.26.5.1170

Old, S. R., \& Naveh-Benjamin, M. (2008). Differential effects of age on item and associative measures of memory: A meta-analysis. Psychology and Aging, 23, 104-108. doi:10.1037/0882-7974.23.1.104

Park, D. C., \& Payer, D. (2006). Working memory across the adult lifespan. In E. Bialystock \& F. I. M. Craik (Eds.), Lifespan Cognition: Mechanisms of change (pp. 128-142). New York, NY: Oxford University Press.

Park, D. C., Polk, T., Park, R., Minear, M., Savage, A., \& Smith, M. (2004). Aging reduces neural specialization in ventral visual cortex. Proceedings of the National Academy of Sciences, USA of the United States of America, 101, 13091-13095. doi:10.1073/pnas.0405148101

Park, D. C., \& Reuter-Lorenz, P. A. (2009). The adaptive brain: Aging and neurocognitive scaffolding. Annual Review of Psychology, 60, 173-196. doi:10.1146/annurev.psych.59.103006.093656

Park, J. Carp, J. Hebrank, A., Park, D. C., \& Polk, T. A. (2010). Neural specificity predicts fluid processing ability in older adults. Journal of Neuroscience, 30, 9253-9259. doi:10.1523/JNEUROSCI.0853-10.2010

Payer, D., Marshuetz, C., Sutton, B., Hebrank, A., Welsh, R., \& Park, D. (2006). Decreased neural specialization in old adults on a working memory task. NeuroReport: For Rapid Communication of Neuroscience Research, 17, 487-491. doi:10.1097/01.wnr.0000209005.40481.31

Pelli, D. (1997). The VideoToolbox software for visual psychophysics: Transforming numbers into movies. Spatial Vision, 10, 437-442. doi: 10.1163/156856897X00366

Phillips, W. A. (1974). Distinction between sensory storage and short-term visual memory. Perception and Psychophysics, 16, 283-290. doi: 10.3758/BF03203943

Reuter-Lorenz, P. A., \& Cappell, K. A. (2008). Neurocognitive aging and the compensation hypothesis. Current Directions in Psychological Science, 17, 177-182. doi:10.1111/j.1467-8721.2008.00570.x

Reynolds, J. H., \& Chelazzi, L. (2004). Attentional modulation of visual processing. Annual Review of Neuroscience, 27, 611-647. doi:10.1146/ annurev.neuro.26.041002.131039

Rouder, J. N., Morey, R. D., Cowan, N., Zwilling, C. E., Morey, C. C., \& Pratte, M. S. (2008). An assessment of fixed-capacity models of visual working memory. Proceedings of the National Academy of Sciences of the United States of America, 105, 5975-5979. doi:10.1073/pnas .0711295105

Sander, M. C., Werkle-Bergner, M., \& Lindenberger, U. (2011). Binding and strategic selection in working memory: A lifespan dissociation. Psychology and Aging, 26, 612-624. doi:10.1037/a0023055

Sara, M., \& Faubert, J. (2000). Aging, perception, and visual short-term memory for luminance-defined form. Ophthalmic \& Physiological Optics, 20, 314-322. doi:10.1046/j.1475-1313.2000.00531.x

Scalf, P. E., \& Beck, D. M. (2010). Competition in visual cortex impedes attention to multiple items. Journal of Neuroscience, 30, 161-169. doi:10.1523/JNEUROSCI.4207-09.2010

Schneider-Garces, N. J., Gordon, B. A., Brumback-Peltz, C. R., Shin, E., Lee, Y., Sutton, B. P., ... Fabiani, M. (2010). Span, CRUNCH, and beyond: Working memory capacity and the aging brain. Journal of Cognitive Neuroscience, 22, 655-669. doi:10.1162/jocn.2009.21230

Shing, Y. L., Werkle-Bergner, M., Brehmer, Y., Mueller, V., Li, S.-C., \& Lindenberger, U. (2010). Episodic memory across the lifespan: The contributions of associative and strategic components. Neuroscience \& Biobehavioral Reviews, 34, 1080-1091. doi:10.1016/j.neubiorev 2009.11.002

Sivia, D. S. (2004). Data analysis: A Bayesian tutorial. Oxford, England Oxford University Press.

Spengler, F., Godde, B., \& Dinse, H. (1995). Effects of ageing on topographic organization of somatosensory cortex. NeuroReport: For Rapid Communication of Neuroscience Research, 6, 469-473. doi:10.1097/ 00001756-199502000-00016

Sweeney, J.A., Rosano, C., Berman, R. A., \& Luna, B. (2001). Inhibitory control of attention declines more than working memory during normal aging. Neurobiology of Aging, 22, 39-47. doi:10.1016/S01974580(00)00175-5

Thurstone, L. L. (1927). Psychophysical analysis. The American Journal of Psychology, 38, 368-389. doi:10.2307/1415006 
Todd, J. J., \& Marois, R. (2004). Capacity limit of visual short-term memory in human posterior parietal cortex. Nature, 428, 751-754. doi: 10.1038 /nature02466

Velhagen, K. (2003). Tafeln zur Prüfung des Farbsinns (33rd ed.). Stuttgart, Germany: Thieme.

Verghese, P. (2001). Visual search and attention: A signal detection theory approach. Neuron, 31, 523-535.

Voss, M. W., Erickson, K. I., Chaddock, L., Prakash, R. S., Colcombe, S. J., Morris, K. S., ... Kramer, A. F. (2008). Dedifferentiation in the visual cortex: An fMRI investigation of individual differences in older adults. Brain Research, 1244, 121-131. doi:10.1016/j.brainres 2008.09.051

Wechsler, D. (1981). Wechsler Adult Intelligence Scale-Revised (WAIS-R). New York, NY: Psychological Corporation.

Welford, A. T. (1981). Signal, noise, performance, and age. Human Factors, 23(1), 97-109.

Welford, A. T. (1984). Between bodily changes and performance: Some possible reasons for slowing with age. Experimental Aging Research, 10, 73-88. doi:10.1080/03610738408258548

Wickens, T. D. (2002). Elementary signal detection theory. Oxford, England: Oxford University Press.

Wilken, P., \& Ma, W. (2004). A detection theory account of change detection. Journal of Vision, 4, 1120-1135. doi:10:1167/4.12.11
Xu, Y., \& Chun, M. (2006). Dissociable neural mechanisms supporting visual short-term memory for objects. Nature, 440, 91-95. doi:10.1038/ nature 04262

Xu, Y., \& Chun, M. M. (2009). Selecting and perceiving multiple visual objects. Trends in Cognitive Sciences, 13, 167-174. doi:10.1016/ j.tics.2009.01.008

Yang, Y., Liang, Z., Li, G., Wang, Y., Zhou, Y., \& Leventhal, A. (2008) Aging affects contrast response functions and adaptation of middle temporal visual area neurons in rhesus monkeys. Neuroscience, 156, 748-757. doi:10.1016/j.neuroscience.2008.08.007

Yang, Y., Zhang, J., Liang, Z., Li, G., Wang, Y., Ma, Y., ... Leventhal A. G. (2009). Aging affects the neural representation of speed in Macaque area MT. Cerebral Cortex, 19, 1957-1967. doi:10.1093/cercor/ bhn221

Yu, S., Wang, Y., Li, X., Zhou, Y., \& Leventhal, A. (2006). Functional degradation of extrastriate visual cortex in senescent rhesus monkeys. Neuroscience, 140, 1023-1029. doi:10.1016/j.neuroscience.2006.01.015

Zhang, W., \& Luck, S. J. (2009). Sudden death and gradual decay in visual working memory. Psychological Sciences, 20, 423-428. doi:10.1111/ j.1467-9280.2009.02322.x

Zhang, W. W., \& Luck, S. J. (2008). Discrete fixed-resolution representations in visual working memory. Nature, 453, 233-235. doi:10.1038/ nature 06860.

\section{Appendix}

\section{Bayesian Inference}

Central to Bayesian inference is the assumption that true parameter values underlying observed data are unknown but can be inferred by integrating our prior knowledge of them and the data we observed. This relationship can be approximated by the proportionality,

$$
p(H \mid D) \propto p(D \mid H) p(H),
$$

where $p(H \mid D)$ is the posterior distribution of the parameter values, $H$, given the observed data, $D, p(H)$ is our prior knowledge about the parameter values, $H$, and $p(D \mid H)$ is the likelihood to observe the data given parameter $H$. In our case, $H=\{\pi, \sigma\}$ is the set of parameters of interest and $D=\left\{(d, N, n)_{I} \mid i=1, \ldots, 6\right\}$ such that at displacement $d_{i}$, we collected a sample of $N_{i}$ Bernoulli trials with $n_{i}$ correct responses. The likelihood of observing the data is given according to our binomial mixture model:

$$
p(D \mid H)=\prod_{i=1}^{6}\left(\begin{array}{c}
N_{i} \\
n_{i}
\end{array}\right) F\left(d_{i}, H\right)^{n_{i}}\left[1-F\left(d_{i}, H\right)\right]^{\left(N_{i}-n_{\mathrm{i}}\right)},
$$

where $F(d, H)$ is the mixture model given in Equation 1. Our prior knowledge or beliefs about the parameters are expressed in terms of the choice of the prior distributions, which is critical because it determines the posterior distribution when the data are not informative. Prior distributions should, therefore, be as specific as possible, but at the same time reflect the level of ignorance sufficiently. Following this rationale, we made the following two decisions. First, the only knowledge we have about the mixture parameter, $\pi$, is that it must take values in the unit interval $[0,1]$. Therefore, we chose a beta distribution $p(\pi \mid 1,1)=\beta(\pi \mid 1,1)$, which is 1 for $0 \leq \pi \geq 1$ and 0 otherwise. Second, the dispersion parameter, $\sigma$, is strictly positive. Therefore, a log-normal distribution could be used. We chose $p(\sigma \mid 1,1)=\log N(\sigma \mid 1,1)$.

Having the priors and the likelihood specified, we sampled from the posterior choosing any combination of $H=\{\pi, \sigma\}$ at random. Markov chain Monte Carlo methods could be used to generate random sequences $H_{1}, H_{2}, \ldots H_{n}$ such that the distribution of $H_{n}$ becomes asymptotical identical to the posterior distribution. We used Hamiltonian sampling implementing the leapfrog method (see Kuss et al., 2005b). In this method, additional sampling parameters, the leapfrog step size and the number of steps, must be specified. We determined these parameters by hand, based on the heuristics proposed by Kuss et al. (2005b).

Received January 14, 2011

Revision received December 15, 2011 Accepted December 15, 2011 